\title{
Erratum zu: Beckmann, M., Mühler, G., Schauenberg, G.: Betriebliche Personalpolitik bei heterogenen Arbeitsmärkten
}

\author{
Der Herausgeber
}

(C) Institut für Arbeitsmarkt- und Berufsforschung 2011

Erratum zu: ZAF (2011) 43: 319-325

DOI 10.1007/s12651-011-0064-8

Der obengenannte Beitrag war für das vorliegende Special Issue vorgesehen, wurde jedoch leider versehentlich bereits im letzten Heft von Band 43 publiziert. Der Vollständigkeit halber wird er hier noch einmal in unveränderter Form abgedruckt.

Wir bitten dieses Versehen zu entschuldigen. 


\title{
Betriebliche Personalpolitik bei heterogenen Arbeitsmärkten
}

\author{
Michael Beckmann · Grit Mühler • Bernd Schauenberg
}

Angenommen: 25. Januar 2011

(C) Institut für Arbeitsmarkt- und Berufsforschung 2011

Zusammenfassung Der Beitrag zeigt auf, welche Flexibilisierungspotentiale sich heutzutage für Unternehmen ergeben, um auf die stetig ansteigenden Anforderungen an alle Arbeitsmarktakteure und die damit verbundene Heterogenisierung des Arbeitsmarkts reagieren zu können. Dabei wird die betriebliche Nachfrage nach zwei Personengruppen, nämlich weiblichen und älteren Arbeitskräften, besonders in den Blick genommen. Unsere Ausführungen fokussieren sich zudem auf zwei unternehmerische Handlungsfelder, die aus unserer Sicht die meisten Flexibilisierungspotentiale bieten: die Weiterqualifikation der Beschäftigten und die Nutzung flexibler Beschäftigungsverhältnisse in Form von Leiharbeit und befristeten Verträgen. Basierend auf Analysen umfangreicher Unternehmens- und Individualdatensätze können wir zeigen, dass die Veränderungen des technologischen und organisatorischen Wandels zum Vorteil von Frauen sowie jüngeren Arbeitnehmern, jedoch zum Nachteil der Männer und älterer Arbeitnehmer

Prof. Dr. M. Beckmann $(\bowtie)$

Wirtschaftswissenschaftliche Fakultät, Abteilung Personal und Organisation, Universität Basel, Peter Merian-Weg 6, 4002 Basel, Schweiz

e-mail: michael.beckmann@unibas.ch

Dr. G. Mühler

Forschungsbereich Arbeitsmärkte, Personalmanagement und Soziale Sicherung, L 7.1, Zentrum für Europäische Wirtschaftsforschung (ZEW), 68161 Mannheim, Deutschland e-mail: muehler@zew.de

Prof. emeritus Dr. Dr. h.c. B. Schauenberg

Wirtschafts- und Verhaltenswissenschaftliche Fakultät,

Betriebswirtschaftliches Seminar III, Personal- und

Organisationsökonomie, Albert-Ludwigs-Universität Freiburg,

Platz der Alten Synagoge, 79085 Freiburg im Breisgau,

Deutschland

e-mail: bernd.schauenberg@vwl.uni-freiburg.de auszufallen scheinen. Der Einsatz von Weiterbildung, befristeten Verträgen und Leiharbeit zeigt positive Effekte, sowohl für die Unternehmen wie auch die Beschäftigten. Die vorgestellten Ergebnisse ermöglichen es Unternehmen, effektiver auf eventuelle demografische und arbeitsangebotsbedingte Engpässe reagieren zu können.

\section{Human resource management in heterogeneous labour markets}

Abstract The objective of this paper is to analyse the flexibility opportunities of firms to respond to increasing labour market requirements and heterogeneity. Thereby, we focus on two groups of workers and two kinds of management events. The two groups of workers are female and older workers, while continuous training and the use of flexible working contracts (i.e. fixed-term employment, temporary agency work) represent our two kinds of management events. On the basis of large individual- and firm-level data sets we find evidence in line with a gender-biased technological and organisational change in favour of female workers and an age-biased technological and organisational change at the expense of older workers. Furthermore, the use of continuous training, fixed-term contracts and temporary agency workers appears to be beneficial for both labour market participants, i.e. firms and workers. Our results enable firms to respond more effectively to labour markets shortages caused by demographic developments or labour supply changes.

\section{Wirtschafts- und unternehmenspolitische Herausforderungen}

Die Rahmenbedingungen innerhalb derer Unternehmen heutzutage agieren haben sich in den letzten zwei Jahrzehn- 
ten deutlich verändert. Die zunehmende Internationalisierung der Faktor- und Absatzmärkte, der technische Fortschritt sowie die Verschiebung der Bedeutung von Wirtschaftssektoren, bewirken Veränderungen in Unternehmensstrategie und Organisationsstruktur und ziehen damit letztlich auch Konsequenzen für die Nachfrage nach und den Umgang mit Arbeitskräften nach sich. Als Folge dieser Entwicklung ist in fast allen entwickelten Industrienationen ein deutlicher Anstieg des formalen Qualifikationsniveaus der Belegschaften in den Betrieben festzustellen (skill upgrading), der von einer Verschiebung der Nachfrage zugunsten qualifizierter Fachkräfte rührt (vgl. z. B. Bound und Johnson 1992; Berman et al. 1998; Machin und Van Reenen 1998; Fitzenberger 1999). Dieser qualifikationsverzerrte technologische Wandel (skill-biased technological change) wird durch einen qualifikationsverzerrten organisatorischen Wandel (skill-biased organisational change) noch verstärkt (Caroli und Van Reenen 2001; Bresnahan et al. 2002). Organisatorische Veränderungen wie das Abflachen von Hierarchien, die Dezentralisierung von Entscheidungen oder die zunehmende Komplexität von Aufgaben stellen steigende Anforderungen an das Qualifikationsprofil der Beschäftigten.

Gleichzeitig nimmt auch beim Arbeitskräfteangebot die Heterogenität zwischen den Beschäftigtengruppen zu. Neben dem in Vollzeit beschäftigten Mann, der nach seiner Berufsausbildung oder dem Studium über viele Jahre beim gleichen Unternehmen arbeitet, haben andere Arbeitnehmergruppen und Beschäftigungsformen an Bedeutung gewonnen. Hierzu zählen nicht nur unterschiedliche Qualifikationen der Arbeitnehmer aufgrund verschiedenartiger Ausbildungen, Fähigkeiten und Arbeitsplatzanforderungen. Die Herausbildung verschiedener Teilarbeitsmärkte kann sich z. B. auch auf eine unterschiedliche Behandlung von männlichen und weiblichen, jüngeren und älteren sowie inländischen und ausländischen Arbeitnehmern beziehen (Fitzenberger et al. 2008).

Die geschilderten Entwicklungen vergrößern den Spielraum für unternehmens- und personalpolitische Entscheidungen, so dass durch die Heterogenisierung neue und flexible Arbeitsvertragsformen (z. B. befristete Arbeitsverträge) und ganz unterschiedliche unternehmenspolitische Strategien, z. B. im Hinblick auf die betriebliche Aus- und Weiterbildung oder die Implementierung von organisatorischen Änderungen und finanziellen Anreizsystemen für die Mitarbeiter, entstehen. Die Determinanten und Folgen personalpolitischer Entscheidungen sind international wie auch national noch nicht hinreichend aufgeklärt. Zudem sind differenziertere Modelle und Forschungsansätze erforderlich Modelle, die die Heterogenität zwischen Beschäftigten einerseits und Unternehmen andererseits adäquat berücksichtigen - um die beobachteten Entwicklungen erklären zu können.
Das Hauptziel dieses Beitrags besteht daher darinaufzuzeigen, wie Unternehmen heute auf die Bedingungen eines ständig steigenden Wettbewerbsdrucks reagieren. Welche Bedeutung hat eine zunehmende Heterogenisierung des Arbeitsmarktes, verursacht durch die stetig ansteigenden Anforderungen an alle beteiligten Akteure auf dem Arbeitsmarkt, für die betriebliche Personalpolitik und welche Flexibilisierungspotentiale lassen sich dadurch erschließen?

Für die Beantwortung dieser Forschungsfrage haben wir zwei Beschäftigtengruppen und zwei Handlungsfelder identifiziert, die nicht nur von hohem wissenschaftlichen Interesse sind, sondern auch eine hohe wirtschaftspolitische Bedeutung und damit auch Politik beratenden Charakter haben. Zunächst richten wir den Fokus auf eine geschlechterund altersspezifische Arbeitsnachfrage und betrachten hierbei mögliche innovationsbedingte Verzerrungen. Des Weiteren analysieren wir den Einsatz von betrieblicher Weiterbildung und die Flexibilisierung von Beschäftigungsverhältnissen durch den Einsatz befristeter Verträge und von Leiharbeit.

Methodisch wurden die genannten Themenbereiche vor allem empirisch-ökonometrisch analysiert. Hierfür konnte auf die Daten von umfangreichen und ergiebigen Betriebsund Individualdatensätzen zurückgegriffen werden. Als Erstes ist hier das Betriebspanel des Instituts für Arbeitsmarktund Berufsforschung (IAB) der Bundesagentur für Arbeit zu nennen. Als zweiten Datensatz verwendeten wir das Sozio-oekonomische Panel des Deutschen Instituts für Wirtschaftsforschung (DIW). Drittens wurde der so genannte LIAB Datensatz eingesetzt, ein linked employer-employeeDatensatz, der über die Verknüpfung des IAB-Betriebspanels mit der Beschäftigtenstatistik zustande kommt.

Der folgende Abschn. 2 betrachtet zunächst die Auswirkungen auf die beiden interessierenden Beschäftigtengruppen, Frauen und ältere Arbeitnehmer, bevor in $\mathrm{Ab}$ schn. 3 unternehmerische Handlungsfelder aufgezeigt werden. Abschnitt 4 fasst die Hauptaussagen kurz zusammen und gibt einen Ausblick auf aktuelle Forschungstrends in der personal- und organisationsökonomischen Forschung.

\section{Betriebliche Arbeitsnachfrage nach qualifizierten Frauen und älteren Arbeitnehmern}

Die einleitenden Ausführungen haben aufgezeigt, dass sich Unternehmen mit ihrer Personalpolitik in einem sehr dynamischen Umfeld bewegen müssen, das insbesondere durch technologische und organisatorische Innovationen geprägt ist. Um den damit einhergehenden Flexibilisierungserfordernissen in personalpolitischer Hinsicht gerecht werden zu können, müssen Betriebe die Qualifikationsstruktur ihrer Belegschaften qualitativ anpassen. Die unmittelbare Folge ist eine zunehmende Heterogenität des Faktors Arbeit. 
Diese Heterogenität konzentriert sich nun allerdings nicht nur auf eine qualifikatorische Differenzierung der Belegschaft. Vielmehr stellt sich auch die Frage nach Entwicklungen in der Beschäftigung weiblicher Arbeitnehmer und in Bezug auf veränderte Altersstrukturen. Der technische Fortschritt könnte also neben qualifikationsverzerrenden Beschäftigungswirkungen auch geschlechts- oder altersspezifische Beschäftigungseffekte nach sich ziehen. Im ersten Fall würden technologische und organisatorische Innovationen die betriebliche Arbeitsnachfrage entweder zugunsten von weiblichen oder zugunsten von männlichen Arbeitskräften verzerren (gender-biased technological and organisational change). Im zweiten Fall hingegen würden Innovationen die Altersstruktur von Belegschaften entweder zugunsten jüngerer oder zugunsten älterer Mitarbeiter beeinflussen (agebiased technological and organisational change).

Die Arbeit von Beckmann et al. (2006) widmet sich dabei der Hypothese eines gender-biased technological and organisational change. Ausgehend von einer produktionstheoretischen Modellierung wird hier empirisch untersucht, ob sich Hinweise auf eine innovationsbedingte Verzerrung der geschlechtsspezifischen Arbeitsnachfrage der Betriebe finden lassen. Als Datenbasis kommen für diese Fragestellung die Wellen der Jahre 2000 bis 2002 des IAB-Betriebspanels zum Einsatz. Technologische Innovationen werden dabei über eine standardisierte Variable erfasst, die sich u. a. aus dem technischen Stand der Anlagen, dem Anteil der betrieblichen Investitionen in Informations- und Kommunikationstechnologien sowie der Verbreitung von Computerarbeitsplätzen im Betrieb zusammensetzt. Organisatorische Innovationen werden ebenfalls mithilfe einer standardisierten Variablen gemessen. In diese Variable werden organisatorische Änderungen aufgenommen, die eine Dezentralisierung von Entscheidungsrechten in den Betrieben abbilden (z. B. Einführung von Profit Centern und Teamarbeit sowie die Delegation von Entscheidungsrechten auf untergeordnete Hierarchieebenen). Für beide Geschlechter werden erweiterte Arbeitsnachfragegleichungen geschätzt, die sich aus einer variablen Translog-Kostenfunktion herleiten lassen. Als Schätzansatz wird zunächst auf die SOLS-Methode (System OLS) zurückgegriffen. Wenn in einem zweiten Schritt zusätzlich eine Analyse für einzelne Qualifikationsstufen innerhalb der beiden Geschlechtergruppen durchgeführt wird, werden für die resultierenden Arbeitsnachfragefunktionen so genannte corner solution models spezifiziert, die dann mit dem Tobit-ML-Verfahren geschätzt werden.

Die empirischen Ergebnisse dieser Studie lassen sich wie folgt zusammenfassen: Die Hypothese eines gender-biased technological change zugunsten weiblicher Arbeitskräfte kann empirisch unterstützt werden. Auch ein skill-biased technological change ist für die Beschäftigung weiblicher und männlicher Arbeitskräfte nachweisbar. Dabei profitieren qualifizierte angestellte Frauen weniger stark von der
Einführung neuer Technologien als ihre männlichen Kollegen. Allerdings sind die Verluste qualifizierter Facharbeiterinnen und unqualifizierter Frauen längst nicht so stark wie in den entsprechenden Kategorien der Männer, was letztendlich auch den gender-biased technological change begründet. Darüber hinaus ergeben sich Hinweise auf einen skillbiased organisational change, aber nicht auf einen genderbiased organisational change.

Die Frage nach der Existenz eines age-biased technological and organisational change kann aufgrund der bisher vorgenommenen empirischen Untersuchungen bejaht werden. Unter Verwendung von Querschnittsdaten des IABBetriebspanels zeigte sich zunächst, dass sowohl technologische als auch organisatorische Innovationen zu steigenden Beschäftigtenanteilen jüngerer Arbeitnehmer (unter 30 Jahre) und zu abnehmenden Beschäftigtenanteilen älterer Arbeitnehmer (ab 50 Jahre) führen (Beckmann 2007). Wir haben für diese Fragestellung aber zusätzlich auch die verknüpften Betriebs- und Beschäftigtendaten (LIAB) verwendet und weiterhin für unbeobachtbare Heterogenität und andere Quellen von Endogenität kontrolliert. Die Verwendung der LIAB-Daten war aus zwei Gründen erforderlich: $\mathrm{Zu}$ nächst einmal konnte mit den LIAB-Daten noch genauer als bisher eine Separierung des ermittelten age-biased technological and organisational change vom skill-biased technological and organisational change erreicht werden. Zweitens konnten mit den LIAB-Daten nun auch Panelanalysen durchgeführt werden, während die Betriebspaneldaten im vorliegenden Fall aufgrund des Umstandes, dass die abhängige Variable einzig in der Welle 1995 enthalten ist, lediglich Querschnittsanalysen zulassen.

Die mithilfe von seemingly unrelated regression (SUR) models für insgesamt neun Alters- und Qualifikationsgruppen erzielten Ergebnisse bestätigen und ergänzen den oben dargestellten altersverzerrenden Effekt. Technische und organisatorische Innovationen begünstigen die Nachfrage nach jüngeren Arbeitnehmern aller Qualifikationsstufen, während die Nachfrage nach älteren Arbeitnehmern mit Ausnahme der hoch qualifizierten älteren Arbeitnehmer zurückgeht (Referenzgruppe: Arbeitnehmer mit mittleren Qualifikationen im mittleren Alter). In der mittleren Altersgruppe steigt die Nachfrage nach hoch qualifizierten Arbeitskräften, während die Nachfrage nach Geringqualifizierten zurückgeht. Mithilfe von System-GMM-Schätzungen wurde in einem weiteren Schritt schließlich potentiellen Verzerrungen aufgrund von unbeobachtbarer Heterogenität sowie umgekehrter Kausalität Rechnung getragen. Die wesentlichen Ergebnisse der SUR-Modelle zum age-biased technological and organisational change bleiben dabei bestehen, fallen aber in etwas abgeschwächter Form aus. Als zentrales Resultat kann also neben der Existenz eines skillbiased technological and organisational change auch ein kausaler age-biased technological and organisational change 
festgestellt werden. Dabei scheint der Alterseffekt den Qualifikationseffekt sogar zu dominieren.

Insgesamt sprechen die Ergebnisse der beschriebenen empirischen Studien zum einen dafür, dass technologische Innovationen, wie z. B. die Verbreitung von Computern in den Unternehmen, die betrieblichen Arbeitsbedingungen in einer Weise verändern, die weiblichen Beschäftigten zugute kommt. Computerarbeitsplätze erfordern weniger Fertigkeiten im physischen Bereich, aber mehr im geistigen Bereich. Somit fällt der komparative Vorteil von männlichen Arbeitskräften bei körperlich anstrengender Arbeit immer weniger ins Gewicht, wenn die Arbeitsplätze einen verstärkten Computereinsatz erfordern. Darüber hinaus konnten unsere Ergebnisse darlegen, dass der technologische und organisatorische Wandel ältere Arbeitskräfte relativ zu anderen Altersgruppen benachteiligt. Damit stehen Unternehmen vor der nicht zu unterschätzenden Herausforderung, technologische und organisatorische Innovationen mit älteren Belegschaften zu bewerkstelligen. Unterstützende Maßnahmen können hierfür u. a. die Etablierung altersgemischter Arbeitsgruppen, eine altersgerechte Anpassung der Arbeitsanforderungen, die Durchführung von Weiterbildungen speziell für ältere Mitarbeiter oder die Rückführung des Senioritätsprinzips sein. Bisherige Untersuchungen des IAB zeigen allerdings, dass derartige Instrumente in deutschen Betrieben bisher nur sehr eingeschränkt eingesetzt werden (Bellmann et al. 2007).

\section{Unternehmerische Handlungsfelder}

Eine Flexibilisierung des Personaleinsatzes kann in Firmen über verschiedene Instrumente erfolgen. Hierzu zählen z. B. betriebliche Qualifizierungsmaßnahmen. Eine andere Möglichkeit ist mit dem Einsatz flexibler Beschäftigungsformen, wie z. B. befristete Beschäftigung, Teilzeitarbeit oder Leiharbeit, gegeben. Beide Handlungsfelder werden im Folgenden näher beschrieben.

\subsection{Flexibilisierungspotentiale durch betriebliche Weiterbildung}

Nach dem Standardmodell der Humankapitaltheorie von Becker (1962) steigen die Löhne von Arbeitnehmern nach Weiterbildungsmaßnahmen aufgrund der damit verbundenen zunehmenden Produktivität, wobei die Lohnzuwächse für allgemein qualifizierte Arbeitnehmer größer sein sollten als für spezifisch qualifizierte Arbeitnehmer. Im Gegensatz zu der überwiegenden Mehrzahl vergleichbarer empirischer Untersuchungen betrachten wir in unserer Arbeit die Lohneffekte der Weiterbildung getrennt für allgemeine und spezifische Weiterbildungsprogramme (Mühler et al. 2007). Zur Anwendung gelangen die Daten des Soziooekonomischen Panels. Als Schätzansätze werden nichtparametrische Matching-Schätzer eingesetzt und mit dem Difference-in-Differences-Schätzer kombiniert, um explizit beobachtbare und unbeobachtbare Individual- und Firmenmerkmale berücksichtigen zu können. Damit ist gewährleistet, dass die erzielten Lohneffekte auch als kausale Effekte interpretiert werden können.

Nach unseren Schätzergebnissen führt allgemeine Weiterbildung zu einer Lohnsteigerung von 5-6 \%, während die Lohneffekte spezifischer Weiterbildungen zwar überwiegend positiv, aber insignifikant sind. Dieses Ergebnis bestätigt damit die Implikationen des Standardmodells der Humankapitaltheorie insoweit, als die Lohnsteigerungen von allgemeiner Weiterbildung größer sind als von spezifischer Weiterbildung. Die Schätzresultate lassen aber noch eine weitere Interpretation zu: Firmen könnten nämlich spezifische Weiterbildung verwenden, wenn eine Anpassung an neue Arbeitsplatzanforderungen vorgenommen werden muss (Anpassungsweiterbildung), während allgemeine Weiterbildung schwerpunktmäßig zur Förderung von Arbeitnehmerkarrieren eingesetzt werden kann, was sich dann auch positiv im Gehalt der Betroffenen bemerkbar macht (Aufstiegsweiterbildung).

Die vorgestellten Ergebnisse ermöglichen es zudem, Unternehmen durch die Ermittlung der Determinanten betrieblicher Weiterbildung einen Handlungsrahmen zur Planung ihrer Qualifizierungsprogramme vorzuschlagen, um dadurch effektiver auf eventuelle demografisch und arbeitsangebotsbedingte Qualifikationsengpässe reagieren zu können.

\subsection{Flexibilisierung von Beschäftigungsverhältnissen}

Ein weiteres bedeutendes Flexibilisierungspotential können sich Unternehmen mit der Kontrahierung so genannter atypischer Beschäftigungsverhältnisse erschließen. Für Deutschland wird immer wieder die vergleichsweise hohe Regulierungsdichte im Zusammenhang mit der Ausgestaltung von Beschäftigungsverhältnissen beklagt. Hier werden vor allem nach unten rigide Lohnstrukturen und die geltenden Kündigungsschutzregeln genannt, die sich in Unternehmensumwelten, die vom technischen und organisatorischen Wandel geprägt sind, als zu starr und nicht mehr zeitgemäß erweisen.

Atypische Beschäftigungsverhältnisse, dazu zählen geringfügige Beschäftigungsverhältnisse (400 $€$-Jobs) sowie Zeitarbeit, Teilzeitarbeit und befristete Arbeitsverträge, scheinen sich in der jüngeren Vergangenheit als persistenter Bestandteil der betrieblichen Personalpolitik etabliert zu haben. Trotzdem ist die Frage noch offen, ob die Stabilität von Beschäftigungsverhältnissen generell abgenommen 
hat (Neumark 2000). Weitere Fragen, für die bisher nur wenig Evidenz vorlag, lauten beispielsweise: Was bewegt Arbeitsanbieter dazu, einen zeitlich befristeten Arbeitsvertrag anzunehmen? Haben die Art und Dauer eines Arbeitsvertrages Auswirkungen auf die Motivation oder Produktivität der Mitarbeiter? Welche Motive über die Verwendung befristeter Verträge herrschen bei den Unternehmen vor? (Vgl. ausführlich hierzu Binz 2007.)

In einer ersten Studie wurde der Frage nachgegangen, ob Arbeitnehmer mit befristetem und unbefristetem Arbeitsvertrag, die unter vergleichbaren organisatorischen Bedingungen arbeiten, Unterschiede in ihrer Arbeitszufriedenheit aufweisen (Beckmann et al. 2009). Derartige Informationen sind für Arbeitgeber sehr hilfreich, weil sie hierdurch einen Eindruck über die Reaktion von heterogenen Arbeitnehmern auf Änderungen in der Arbeitsorganisation gewinnen. Überraschenderweise lautet ein zentrales Ergebnis, dass Arbeitnehmer mit befristetem Arbeitsvertrag in vielen organisatorischen Situationen zufriedener mit ihrer Tätigkeit sind als unbefristet Beschäftigte.

Für diese Fragestellung wurden die Daten des Soziooekonomischen Panels herangezogen. Als Schätzmethoden kommen zunächst lineare Fixed effects-Modelle zum Einsatz, die in einem weiteren Schritt mit einem zweistufigen Schätzansatz nach Dubin und McFadden (1984) kombiniert werden, um neben unbeobachtbaren Arbeitnehmercharakteristika zusätzlich für andere Endogenitätsquellen zu kontrollieren. Die Resultate belegen, dass Arbeitnehmer mit befristeten und unbefristeten Verträgen im Hinblick auf ihre Arbeitszufriedenheit durchaus unterschiedlich reagieren, wenn sie mit vergleichbaren Situationen konfrontiert sind. Das gilt z. B. für arbeitsorganisatorische Merkmale, wie Aufgabenvielfalt, Mitbestimmung oder die sozialen Beziehungen zu Vorgesetzten und Untergebenen.

Ein weiterer Beitrag von Beckmann und Kuhn (2009) untersucht zum einen die Frage, welche Produktivitätseffekte beim Einsatz von Leiharbeit festzustellen sind. Zum anderen wird darin getestet, ob die betriebliche Strategie, die hinter dem Einsatz von Leiharbeit steckt, einen Produktivitätseffekt hat. Zum Einsatz kommen jeweils die Daten des IABBetriebspanels (Wellen 2002 bis 2005). Für die erste Fragestellung wird eine Cobb-Douglas-Produktionsfunktion spezifiziert, die um eine Variable für den Anteil der Leiharbeiter (in originärer und quadrierter Form) sowie weitere Kontrollvariablen erweitert wird. Geschätzt werden gepoolte OLSModelle (als Referenzansatz), Random und Fixed effectsModelle (zur Kontrolle unbeobachtbarer Firmenmerkmale) sowie Panel-IV-Modelle (zur Kontrolle verbleibender Endogenität aufgrund von umgekehrter Kausalität). Für die zweite Fragestellung wird aufgrund der Datenverfügbarkeit (die Variablen zur Erfassung der Leiharbeitsstrategien sind nur in einer Welle enthalten) ein zweistufiger Schätzansatz nach Black und Lynch (2001) angewendet, um für unbeobachtbare Heterogenität zu kontrollieren. Potentiell verbleibende
Endogenitätsprobleme werden schließlich durch die Kombination des Black und Lynch-Ansatzes mit dem Korrekturverfahren nach Dubin und McFadden (1984) berücksichtigt.

Im Hinblick auf die erste Fragestellung sprechen die empirischen Ergebnisse für einen umgekehrt u-förmigen Produktivitätsverlauf bei einem zunehmenden Einsatz von Leiharbeit. Demnach erweist sich der betriebliche Einsatz von Leiharbeitern im Grundsatz als produktivitätsfördernd. Erst für relativ hohe Anteile an Leiharbeitern (mehr als $25 \%)$ sind wieder Produktivitätsverluste zu verzeichnen. Im Hinblick auf die zweite Fragestellung konnte festgestellt werden, dass Betriebe, die beim Einsatz von Leiharbeit eine Screening-Strategie verwenden (d. h. Leiharbeiter werden nach Bewährung und bei Bedarf vom Betrieb übernommen), signifikant produktiver sind als Betriebe mit einer Flexibilisierungsstrategie (Leiharbeiter werden lediglich beschäftigt, um Nachfrageschwankungen auszugleichen). Es ist daher für Unternehmen, die Leiharbeiter einsetzen, in ihrem eigenen Interesse, diesen eine längerfristige Perspektive im Betrieb zu ermöglichen.

Insgesamt zeigen unsere Arbeiten zum betrieblichen Einsatz flexibler Beschäftigungsverhältnisse demnach vor allem positive Effekte. Das gilt sowohl für die Unternehmen als auch für die betroffenen Arbeitnehmer.

\section{Zusammenfassung und Ausblick}

Die zentrale Zielsetzung des Forschungsprojektes, das diesem Beitrag zugrunde liegt, bestand in der Auseinandersetzung mit der Frage, wie die betriebliche Personalpolitik auf die gravierenden Herausforderungen reagieren kann, die sich z. B. durch technologische und organisatorische Innovationen aber auch durch die seit geraumer Zeit zu beobachtenden arbeitsangebotsseitigen Effekte ergeben. Wir haben uns zur Beantwortung dieser Forschungsfrage auf zwei Beschäftigtengruppen und zwei Handlungsfelder konzentriert, die uns nicht nur wissenschaftlich interessant erschienen, sondern auch eine große arbeitmarktpolitische Bedeutung haben. Zunächst haben wir unsere Aufmerksamkeit auf eine geschlechter- und altersspezifische Arbeitsnachfrage gerichtet und hierbei mögliche innovationsbedingte Verzerrungen betrachtet. Im Hinblick auf die zwei ausgewählten betrieblichen Handlungsfelder haben wir zum einen den Einsatz betrieblicher Weiterbildung und zum anderen die Flexibilisierung von Beschäftigungsverhältnissen in Form der Verwendung befristeter Arbeitsverträge sowie des Einsatzes von Leiharbeitnehmern analysiert.

Ein wesentliches Ziel unserer Analysen bestand bei allen Themenschwerpunkten auch in der Ableitung konkreter Gestaltungsempfehlungen für die betriebliche Personalpolitik. Das wird beispielsweise deutlich, wenn wir bei der Betrachtung der Auswirkungen von Innovationen auf die be- 
triebliche Altersstruktur als Konsequenz aus dem empirischen Befund potentielle Anpassungsstrategien der Betriebe zur Vereinbarkeit von Innovationen und der Beschäftigung älterer Arbeitnehmer diskutieren oder geeignete Weiterbildungsmaßnahmen für ältere Arbeitnehmer empfehlen. Weiterhin kann den Unternehmen durch die Ermittlung der Determinanten und Lohneffekte betrieblicher Weiterbildung ein Handlungsrahmen zur Planung ihrer Qualifizierungsprogramme vorgeschlagen werden, damit diese effektiver auf eventuelle demografisch und arbeitsangebotsbedingte Qualifikationsengpässe reagieren können.

Die Studien zu den Effekten flexibler Beschäftigungsformen auf die Arbeitszufriedenheit der betroffenen Arbeitnehmer, auf Mitarbeitermotivation und Produktivität sollen unmittelbar Aufschluss über die Möglichkeiten und Grenzen des betrieblichen Einsatzes derartiger Personalinstrumente geben. Schließlich haben wir in weiteren Forschungsarbeiten, die hier aus Platzgründen nicht umfassend diskutiert werden konnten, unmittelbar auf betriebliche Personalstrategien abgestellt, indem wir nach Komplementaritäten zwischen personalpolitischen Maßnahmen fragen und damit die Auswirkungen von ganzen Bündeln bzw. Systemen an Personalanpassungsmaßnahmen auf den Unternehmenserfolg untersuchen (Teuber 2008).

Sämtliche in diesem Beitrag bisher vorgestellten empirischen Studien, die im Verlauf des DFG-Schwerpunktprogramms durchgeführt wurden, verwenden etablierte Massendatensätze auf der individuellen bzw. betrieblichen Ebene. Als Alternative zu der Arbeit mit großen repräsentativen Datensätzen hat sich in der Zwischenzeit jedoch auch eine Forschungsmethode entwickelt, die oftmals als ,insider econometrics" bezeichnet wird (vgl. u. a. Shaw 2009). Hierbei werden die Wirkungen unternehmerischer Personalmaßnahmen anhand der Daten einzelner Unternehmen analysiert. Ein Vorteil im Vergleich zu Datensätzen mit Beobachtungen zahlreicher Unternehmen besteht beispielsweise darin, dass eine besonders detaillierte Messung der gewünschten Maßnahmen möglich wird. So können etwa Weiterbildungsdauern präzise in Tagen und Stunden, die Inhalte und Art der jeweiligen Maßnahme sowie die möglichen Folgen (z. B. Lohnerhöhung, Beförderung, Weiterbeschäftigung) erfasst werden, anstatt auf dichotome Abfragen zurückzugreifen, die in den alternativen Unternehmens- oder Individualdatensätzen typischerweise verwendet werden. Dadurch werden mikroökonomische Analysen zu Fragestellungen möglich, die mit den üblichen Informationen aus Survey- und administrativen Datensätzen nicht zu beantworten wären. Weiterhin werden durch die Nutzung der Daten von einzelnen Unternehmen Heterogenitäten im Arbeitsumfeld minimiert, die beispielsweise aus der Branchenzugehörigkeit, den beschäftigten Berufsgruppen oder den Unternehmenszielen resultieren. Als Folge daraus lassen sich Effekte extrahieren, welche z. B. die Wirkung von Management-Maßnahmen auf die Produktivität sauber erfassen.
Auch in Deutschland kann in Einzelfällen inzwischen auf derartige Unternehmensdaten zurückgegriffen werden. Damit wird es nun möglich, Erkenntnisse zu den Effekten solcher Maßnahmen zu erlangen, die anderenfalls aus Mangel an Beobachtbarkeit eine „black box“ bleiben würden. Auch im Rahmen des DFG-Schwerpunktprogramms ist mit den Personaldaten eines deutschen Großunternehmens zu Fragestellungen der betrieblichen Weiterbildung gearbeitet worden. Die Ergebnisse dieser Untersuchungen, die hier aus Platzgründen nicht näher erläutert werden können, finden sich in Mühler (2010). Ähnlich wie die Realisierung experimenteller Studien scheint sich somit auch die Auswertung von Einzelunternehmensdaten zu einer vielversprechenden Ergänzung der empirischen Arbeiten mit großen repräsentativen Datensätzen zu entwickeln.

\section{Literatur}

Becker, G.S.: Investment in human capital: a theoretical analysis. J. Polit. Econ. 70, 9-49 (1962)

Beckmann, M.: Age-biased technological and organizational change: firm-level evidence and management implications. WWZdiscussion paper 05/07, WWZ-Center of Business and Economics, University of Basel (2007)

Beckmann, M., Kuhn, D.: Temporary agency work and firm performance: evidence from German establishment-level panel data. GEABA discussion paper No. 09-17, German Economic Association of Business Administration (2009)

Beckmann, M., Cornelissen, T., Schauenberg, B.: Fixed-term employment, work organization and job satisfaction: evidence from German individual-level data. WWZ-discussion paper 08/09, WWZ - Center of Business and Economics, University of Basel (2009)

Beckmann, M., Schauenberg, B., Timmermann, A.: Betriebliche Innovationen und geschlechterspezifische Arbeitsnachfrage. Die Betriebswirtschaft 66, 287-306 (2006)

Bellmann, L., Kistler, E., Wahse, J.: Betriebe müssen sich auf alternde Belegschaften einstellen. IAB Kurzbericht Nr. 21 (2007)

Berman, E., Bound, J., Machin, S.: Implications of skill-biased technological change: international evidence. Q. J. Econ. 113, 12451279 (1998)

Binz, A.: Einflussfaktoren und Erfolgswirkungen befristeter Beschäftigungsverhältnisse. Hamburg (2007)

Black, S.E., Lynch, L.M.: How to compete: the impact of workplace practices and information technology on productivity. Rev. Econ. Stat. 83, 434-445 (2001)

Bound, J., Johnson, G.: Changes in the structure of wages in the 1980's: an evaluation of alternative explanations. Am. Econ. Rev. 82, 371-392 (1992)

Bresnahan, T., Brynjolfsson, E., Hitt, L.: Information technology, workplace organization and the demand for skilled labor: firmlevel evidence. Q. J. Econ. 117, 339-376 (2002)

Caroli, E., Van Reenen, J.: Skill biased organizational change? Evidence from a panel of British and French establishments. Q. J. Econ. 116, 1449-1492 (2001)

Dubin, J.A., McFadden, D.L.: An econometric analysis of residential electric appliance holdings and consumption. Econometrica 52, 345-362 (1984)

Fitzenberger, B.: Wages and Employment Across Skill Groups: An Analysis for West Germany. Physica-Verlag, Heidelberg (1999) 
Fitzenberger, B., Hübler, O., Kraft, K.: Flexibilisierungspotenziale bei heterogenen Arbeitsmärkten - Eine Einführung. Z. Arbeitsmarktforsch. 2/3, 95-116 (2008)

Machin, S., Van Reenen, J.: Technology and changes in skill structure: evidence from seven OECD countries. Q. J. Econ. 113, 12151244 (1998)

Mühler, G.: Five essays on early education and workplace training. Dissertation, Albert-Ludwigs-Universität Freiburg (2010)

Mühler, G., Beckmann, M., Schauenberg, B.: The returns to continuous training in Germany: new evidence from propensity score matching estimators. Rev. Manage. Sci. 1, 209-235 (2007)

Neumark, D.: Changes in the job stability and job security: a collective effort to untangle, reconcile, and interpret the evidence. NBER working paper No. 7472, National Bureau of Economic Research, Cambridge (2000)

Shaw, K.: Insider econometrics: a roadmap with stops along the way. Labour Econ. 16, 607-617 (2009)

Teuber, S.: Continuing vocational (firm-specific) training and human resource management practices - a complementary relationship? Evidence from IAB establishment panel. Mimeo (2008)

Prof. Dr. Michael Beckmann Studium der Wirtschaftswissenschaften an der Universität Hannover, 1993 Abschluss als Diplom-Ökonom, 1993-2004 wissenschaftlicher Mitarbeiter und Assistent an den Universitäten Würzburg und Freiburg, 1998 Promotion zum Dr. rer. pol. und 2004 Habilitation an der Universität Freiburg, 2004-2007 Universitätsprofessor für Wirtschaftspädagogik, insbesondere Betriebspädagogik an der Ludwig-Maximilians-Universität München, seit 2007
Ordinarius für Personal und Organisation an der Universität Basel. Forschungsfelder: Personalökonomie, Organisationsökonomie. E-Mail: michael.beckmann@unibas.ch.

Dr. Grit Mühler Studium der Betriebswirtschaftslehre an der Universität Mannheim, 2005 Abschluss als Diplom-Kauffrau, 2005-2006 wissenschaftliche Mitarbeiterin am Deutschen Institut für Internationale Pädagogische Forschung (DIPF) in Frankfurt am Main, seit 2006 wissenschaftliche Mitarbeiterin am Zentrum für Europäische Wirtschaftsforschung (ZEW) im Bereich „Arbeitsmärkte, Personalmanagement und Soziale Sicherung“, 2010 Promotion zum Dr. rer. pol. an der Universität Freiburg. Forschungsfelder: Bildungsökonomie, Personalökonomie. E-Mail: mühler@zew.de.

Prof. emeritus Dr. Dr. h.c. Bernd Schauenberg Studium der Betriebswirtschaftslehre an der Universität Frankfurt am Main, 1971 Abschluss als Diplom-Kaufmann, 1972-1977 wissenschaftlicher Mitarbeiter an der Universität Frankfurt am Main, 1976 Promotion zum Dr. rer. pol., 1977-1979 Habilitationsstipendium (DFG), 1980-1985 Hochschulassistent an der Universität Frankfurt am Main, 1983 Habilitation, 1985-1986 Dozent an der European Business School, 1986-1988 Professor für Betriebswirtschaftslehre an der Universität Hannover, 1988-1992 Professor für Betriebswirtschaftslehre an der Freien Universität Berlin, 1992-1997 Professor für Betriebswirtschaftslehre an der Universität Würzburg, 1997-2010 Professor für Betriebswirtschaftslehre an der Universität Freiburg, 2009 Dr. h.c. Universität Paderborn. Forschungsfelder: Personalökonomie, Organisationsökonomie, Theorie der Unternehmung. E-Mail: bernd.schauenberg@vwl.uni-freiburg.de. 